\title{
1 Understanding the diversity of DNA methylation in Mycobacterium
} 2 tuberculosis

Authors:

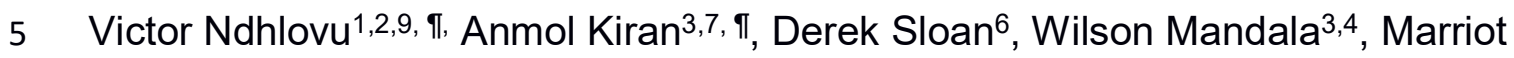

6 Nliwasa ${ }^{2, .9}$, Dean B Everett ${ }^{7}$, Mphatso Mwapasa ${ }^{9}$, Konstantina Kontogianni ${ }^{5}$, Mercy

7 Kamdolozi², Elizabeth L Corbett $3,9,10$, Maxine Caws ${ }^{5,8}$, Gerry Davies ${ }^{1,3}$

\section{Affiliations}

1. University of Liverpool, Liverpool, UK

2. University of Malawi, College of Medicine, Biomedical Sciences Department, Blantyre, Malawi

3. Malawi-Liverpool Wellcome Trust, Blantyre, Malawi

4. Academy of Medical Sciences, Malawi University of Science and Technology (MUST), Thyolo, Malawi

5. Liverpool School of Tropical Medicine, Liverpool, UK

6. University of Saint Andrews, UK

7. Edinburgh University, Edinburgh, UK

8. Birat Nepal Medical Trust, Lazimpat, Kathmandu.

9. Helse Nord Tuberculosis Initiative Project, University of Malawi, College of Medicine, Blantyre, Malawi.

10. London School of Hygiene \& Tropical Medicine (LSHTM), London, United Kingdom.

IThese authors contributed equally as first authors.

\section{Corresponding Author:}

Email: vndhlovu@medcol.mw (VN) 


\section{Abstract}

Although Mycobacterium tuberculosis (Mtb) strains exhibit genomic homology of $>99 \%$, there is considerable variation in the phenotype. The underlying mechanisms of phenotypic heterogeneity in Mtb are not well understood but epigenetic variation is thought to contribute. At present the methylome of Mtb has not been completely characterized. We completed methylomes of 18 Mycobacterium tuberculosis (Mtb) clinical isolates from Malawi representing the largest number of Mtb genomes to be completed in a single study using Single Molecule Real Time (SMRT) sequencing to date. We replicate and confirm four methylation disrupting mutations in lineages of $M t b$. For the first time we report complete loss of methylation courtesy of C758T (S253L) mutation in the MamB gene of Indo-oceanic lineage of $M t b$. We also conducted a genomic and methylome comparison of the Malawian samples against a global sample. We confirm that methylation in Mtb is lineage specific although some unresolved issues still remain.

\section{Introduction}

Tuberculosis (TB) is a disease that remains a global health crisis with an estimated 1.7 billion people infected of which $5-10 \%$ will develop the disease in their lifetime (WHO, 2020). The major barriers to disease elimination have been lack of an effective vaccine or fast and effective diagnostic tools, increasing drug resistance and co-infection with HIV (Davies et al., 2014; De Schacht et al., 2019). Mycobacterium tuberculosis (Mtb), the causative agent of TB, has a genome with a uniformly high guanine + cytosine $(65.6 \%)$ owing to minimal incorporation of foreign DNA during its evolution (Cole, 1999). One 
unique feature of the Mtb genome is the large number of genes it contains. Up to $10 \%$ of the total coding potential contains polymorphic guanine-cytosine repetitive sequences (PGRS) (Cole, 2002; Grover et al., 2018) which encode two unrelated families of acidic glycine-rich proteins- proline-glutamic acid (PE) and proline-proline glutamic acid (PPE). Specific functions of these genes and their proteins remain unclear (Cole, 2002; Fishbein et al., 2015; J E Phelan et al., 2016) although they have been implicated in immune evasion and virulence (Fishbein et al., 2015; J E Phelan et al., 2016) . Consistently, evidence has suggested that proteins located in the cell wall and cell membranes are responsible for diversity in antigenic structure and virulence. This greatly contributes to Mtb evolution and adaptation to different hosts (Brennan \& Delogu, 2002; Filliol et al., 2006). Although Mtb strains have been shown to exhibit genomic homology of $>99 \%$ (Hershberg et al., 2008) such similarity is rarely replicated in the phenotype. This phenotypic heterogeneity has been seen in the virulence of the Beijing strain which has been associated with increasing multidrug resistant TB (MDR-TB) (Cowley et al., 2008; van der Spuy et al., 2009) whereas the East African Indian (EAI) lineage has been associated with lower rates of transmission compared to other lineages (Albanna et al., 2011). Similarly, the Euro-American lineage is the most geographically successful strain (Gagneux \& Small, 2007) but specific mechanisms supporting this successful dissemination remain unknown. Phenotypic heterogeneity in Mtb has been associated with epigenetic inheritance (Balaban et al., 2004) and the most common epigenetic mechanism in

Mtb is DNA methylation (Casadesus \& Low, 2006; Shell et al., 2013). A few studies have characterized the $M t b$ methylome and revealed three 6-methyladenine (m6A) motifs and 
their cognate methyltransferases (Mtases), MamA, MamB and HsdM respectively (Shell et al., 2013; Zhu et al., 2015). Using Pacific Biosciences Single Molecule Real Time (SMRT) sequencing, two studies have recently shown that specific mutations in the Mtases lead to loss of Mtase activity and may play a role in evolution of Mtb (J. Phelan et al., 2018; Zhu et al., 2015). At present the methylome of Mtb has not been completely characterized, neither has any resulting information been correlated with phenotypic heterogeneity observed in TB patients. Understanding the complete biology of Mtb will aid in developing strategies for reducing the Mtb treatment duration from the standard 6 months.

We present characterization of methylomes of 18 Mycobacterium tuberculosis (Mtb) isolates from patients in Blantyre, Malawi including 12 Euro-American lineage (L4) strains, the most prevalent phylogenetic lineage in Malawi, 3 Beijing lineage strains (L2) and 3 Indo-oceanic lineage (L1) strains. This work presents the largest number of $M t b$ genomes of a single lineage to be completed in a single study using Single Molecule Real Time (SMRT) sequencing to date. Additionally, we confirm three confident sequence motifs in Mtb and confirm the strain specific mutations responsible for loss of methyltransferase activity in Mtb. Additionally, for the first time we report the complete loss of methylation courtesy of a novel mutation C758T (S253L) in Indo-oceanic lineage (L1). Through a genomic and methylome comparative analysis with a global sample of 16 samples we report previously unreported mutation affecting the pks15/1 locus in L6 and L6 isolates. 


\section{Results}

\section{Lineage Analysis of Mycobacterium tuberculosis}

Experimental (RD-PCR) and computational (TB-Profiler) outcomes on Malawian strains lineage identification were consistent as: 3/18 (17\%) were L1 (Indo-Oceanic), 3/18 (17\%) were L2 (East-Asian) and 12/18 (66\%) were L4 (Euro-American). De novo reporting of global sample lineages (J. Phelan et al., 2018) (16 samples) using TB-Profiler was as follows : 3/16 L1(Indo-oceanic), 2/16 L2 (East-Asian), 3/16 L4 (Euro-American), 2/16 L5 (West African 1 and 6/16 L6 (West African 2) (Table 1). Using a reference with an intact pks15 (Rv2947c) gene, it was possible to identify the $15 / 34$ strains belonging to L4 in the combined dataset. These possessed a 7 bp deletion (GGGCCGC) in the pks15/1 gene as previously documented (Constant et al., 2002; Gagneux \& Small, 2007). Additionally, pks15 (Rv2947c) could be used to assign lineages to the rest of the samples. All L1 (6/34 strains) had a G1318C substitution and GGGCCGC insertion while L2 (5/34) strains had a GGGCCGC insertion only. All L5 samples had a 9bp deletion (CGGTGCTGG,10971105), a distinct substitution A50G and an insertion GGGCCGC. A L1, L5, L6 (1318 G>C substitution) and a L6 (1658 1bp insertion of G), L1, L2, L5 (1658 7bp insertion) (Fig 1)

(1)


116 Table 1: Lineages and sub-lineages of the samples reported by TB-Profiler using assembled genomic sequences (ERS-Malawian and SAMEA-global samples).

\begin{tabular}{|c|c|c|c|}
\hline Sample ID & Lineage & Sub-lineage & Sub-sub-lineage \\
\hline ERS2711939 & Lineage4 & Lineage4.3 & Lineage4.3.4 \\
\hline ERS2711940 & Lineage4 & Lineage 4.3 & Lineage4.3.4 \\
\hline ERS2711941 & Lineage4 & Lineage 4.3 & \\
\hline ERS2711942 & Lineage4 & Lineage 4.3 & Lineage4.3.4 \\
\hline ERS2711943 & Lineage1 & Lineage1.1 & Lineage1.1.3 \\
\hline ERS2711944 & Lineage4 & Lineage 4.3 & Lineage4.3.4 \\
\hline ERS2711945 & Lineage4 & Lineage 4.3 & Lineage4.3.4 \\
\hline ERS2711946 & Lineage4 & Lineage4.3 & Lineage4.3.4 \\
\hline ERS2711947 & Lineage4 & Lineage4.3 & Lineage4.3.4 \\
\hline ERS2711948 & Lineage1 & Lineage1.1 & Lineage1.1.3 \\
\hline ERS2711949 & Lineage4 & Lineage4.3 & Lineage4.3.4 \\
\hline ERS2711950 & Lineage4 & Lineage4.5 & \\
\hline ERS2711951 & Lineage4 & & Lineage4.1.2 \\
\hline ERS2711952 & Lineage4 & Lineage4.3 & Lineage4.3.4 \\
\hline ERS2711953 & Lineage2 & Lineage2.2 & \\
\hline ERS2711954 & Lineage2 & Lineage 2.2 & \\
\hline ERS2711955 & Lineage2 & Lineage2.2 & \\
\hline ERS2711956 & Lineage1 & Lineage1.1 & Lineage1.1.3 \\
\hline SAMEA104606019 & Lineage1 & Lineage1.1 & Lineage1.1.3 \\
\hline SAMEA104606020 & Lineage1 & Lineage1.1 & Lineage1.1.3 \\
\hline SAMEA104606021 & Lineage1 & Lineage1.1 & Lineage1.1.3 \\
\hline SAMEA104606022 & Lineage5 & & \\
\hline SAMEA104606023 & Lineage2 & Lineage2.2 & Lineage2.2.1 \\
\hline SAMEA104606024 & Lineage4 & Lineage4.3 & Lineage4.3.4 \\
\hline SAMEA104606025 & Lineage4 & Lineage4.1 & Lineage4.1.2 \\
\hline SAMEA104606026 & Lineage6 & & \\
\hline SAMEA104606027 & Lineage6 & & \\
\hline SAMEA104606028 & Lineage2 & Lineage2.2 & Lineage2.2.1 \\
\hline SAMEA104606029 & Lineage4 & Lineage4.3 & Lineage4.3.4 \\
\hline SAMEA104606030 & Lineage5 & & \\
\hline SAMEA104606031 & Lineage6 & & \\
\hline SAMEA104606032 & Lineage6 & & \\
\hline SAMEA104606033 & Lineage6 & & \\
\hline SAMEA104606034 & Lineage6 & & \\
\hline
\end{tabular}




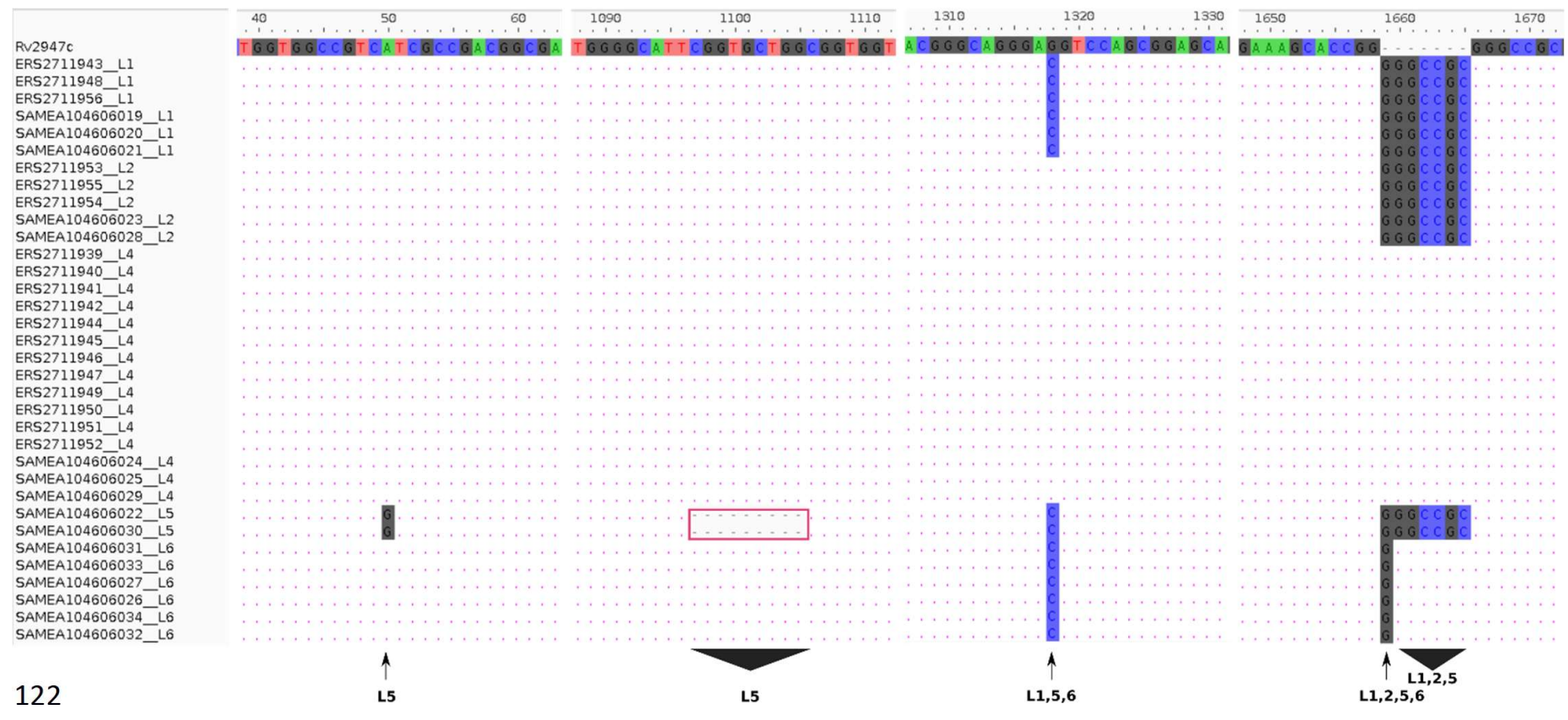

123 Figure 1: Lineage specific sequence differences relative to the reference gene 124 pks15 (Rv2947c)

125 The pks15 gene from 34 samples was aligned against the reference to display lineage

126 specific variations. Variants were observed in four different locations/ranges within the

127 gene discriminating four lineages L5 (50, A>G substitution), L 5 (1097-1105

128 CGGTGCTGG deletion), L1, L5, L6 (1318 G>C substitution) and L6 (1658 1 bp insertion 129 of G), L1, L2, L5 (1658 7bp insertion)

\section{DNA Methylation Patterns}

132 The m6A methylation motifs present in more than 10 isolates were CACGCAG (820

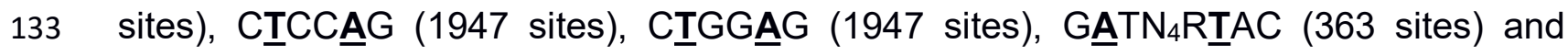

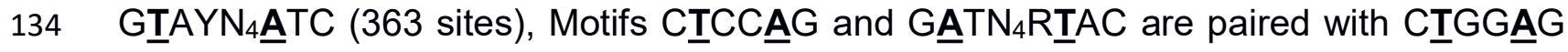

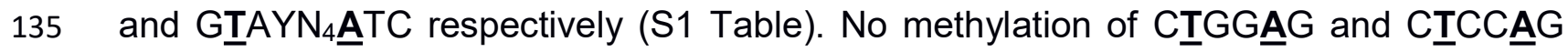
136 was reported in all L2 samples including one L6 sample (SAMEA104606027). One 137 sample from L4 (ERS2711941) lacked methylation in CICCAG motif (Fig 2A). These 
motifs are methylated by mamA Mtase (Shell et al., 2013). Multiple sequence comparison with the reference gene (Rv3263) revealed that all L2 samples had a A809C change resulting in E270A as previously reported (J. Phelan et al., 2018; Shell et al., 2013; Zhu et al., 2015) (including G1199C, W400S in two samples only). Consequently, nonmethylated L6 samples had an alteration at A1378G resulting in A460T in Rv3263. The L4 sample showing no methylation in motif CICCAG had a synonymous substitution at C216T and a non-synonymous substitution at G454A resulting in G152S amino acid substitution. To our knowledge, this potentially methylation disrupting mutation has not been previously reported. The motif CACGCAG is methylated by the mamB Mtase (J. Phelan et al., 2018; Zhu et al., 2015). Two of the six L1 samples (ERS2711948, ERS2711956) lacked this methylation (Fig 2A). Methylation in the rest of the samples was however below $80 \%$ (range 56\% - 79.6\%) (Fig 2C). Surprisingly, all L1 samples (6) possessed a C758T resulting in amino acid change S253L in the mamB gene (Rv2024c). This mutation has previously been reported to be responsible for partial loss of methylation in L1 samples (J. Phelan et al., 2018). This is the first time that mutation S253L is being associated with complete loss of MamB Mtase. Lineage 2 sample (ERS2711953) and L4 sample (ERS2711945) had low methylation in motif CACGCAG (65\% and $73 \%$ ) compared to other samples from the same lineage (100\%) but these samples had no specific mutations in the mamB gene. No effect of L6 specific mutation $\mathrm{R} 289 \mathrm{C}$ and $\mathrm{L} 5$ specific mutation $\mathrm{L} 452 \mathrm{~V}$ was observed on the mamB methylation in these lineages (Fig 2C). However, non-lineage specific multiple variation was reported at 3' end. Motifs GATN 4 RIAC (363 sites) and GIAYN 4 ATTC (363 sites) are methylated by $h s d M$ (Rv2756c) and hsdS (Rv2761c) genes (J. Phelan et al., 2018; Zhu et al., 2015) . One L1 
sample (ERS2711956) showed no methylation in either motif however, ERS2711948 was methylated at GIAY $\mathrm{N}_{4} \underline{\mathbf{A}}$ TC only (Fig 2A). The $h s d M$ gene sequences were identical for all L1 samples and no 5'-upstream alterations (300bp) were reported either. All the L4

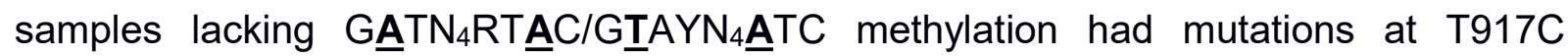
resulting in L306P in $h s d M$ gene and G74T resulting in G25V amino acid change in $h s d S$ gene. While the T917C (L306P) was previously characterized (J. Phelan et al., 2018; Shell et al., 2013; Zhu et al., 2015)., the G74T(G25V) mutation in hsdS gene has not been previously reported. The distribution of lineages specific motif methylation is show in Fig 2B and Fig 2D.
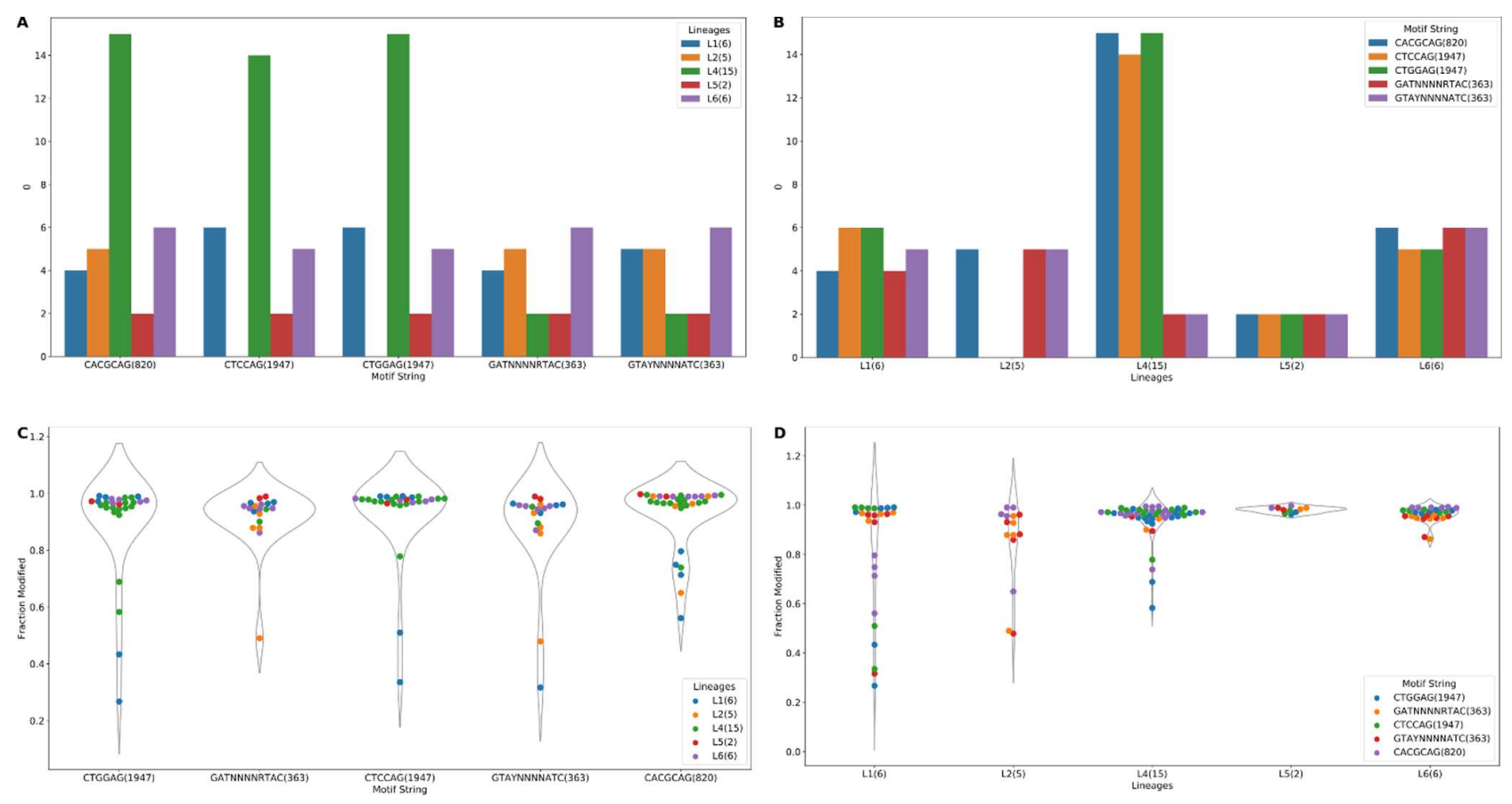

\section{Figure 2: Methylation summary}

(A) Distribution of methylated samples in each Lineage for the motifs. (B) Distribution of samples with methylated motifs in each lineage. (C) Methylation efficiency in samples for each motif. (D) Methylation efficiency by motif in each lineage. 


\section{Methylation Efficiency among lineages}

Among the samples having methylation in major motifs, most reported methylation efficiency was higher than 82\% (Fig 2C, S1 Table). Lineage 4 sample (ERS2711941) had $58 \%$ methylation for CICCAG and it lacked methylation on the CIGGAG motif while another L4 sample (ERS2711945) had 69\% and 79\% methylation on CIGGAG and CICCAG respectively. Two L1 samples (ERS2711948, ERS2711956) showing methylation of $27 \%$ and $36 \%, 43 \%$ and $51 \%$ for CIGGAG and CICCAG respectively but having no specific mutation in methylation conferring genes. Methylation distribution within motifs for each sample is displayed in Fig 2D. Lineage 1 sample (ERS2711948) was methylated at $32 \%$ on motif GIAYN 4 ATC, while L2 sample (ERS2711953) was methylated at $48 \%$ on this motif and $49 \%$ on motif GATN ${ }_{4}$ RIAC. Other samples with low efficiency were as follows: ERS2711953 from L2 with 65\% methylation efficiency and L1 samples SAMEA104606020, SAMEA104606019, SAMEA104606021 and ERS2711943 with $71 \%, 80 \%, 75 \%$ and $56 \%$ respectively on CACGCAG (Fig $2 \mathrm{C}$ and Fig 2D).

\section{Comparison of Methylation within Mtb strains}

The strain arrangements in the m6A IPD ratio based cladogram clusters and genome based maximum likelihood (ML) phylogeny were compared (Fig 3). The samples clustered into four IPD based groups. However, in the ML phylogeny lineages formed distinct clusters. Lineage 2 samples and one L6 sample (SAMEA104606027) with no CIGGAG and CICCAG methylation clustered together. Two samples belonging to L4 (ERS2711951, SAMEA104606025) having no methylation in GATN 4 RIAC and GIAYN 4 ATC motifs clustered with L5 and L6 samples in the IPD ratio cladogram (Fig 3). 


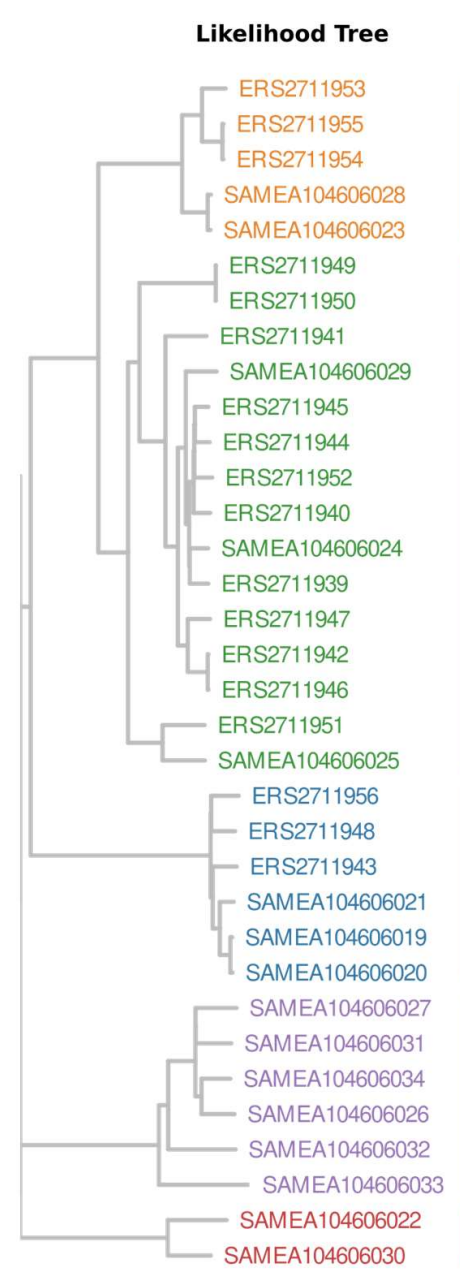

\section{$\mathbf{m}^{6} \mathrm{~A}$ Clutering}
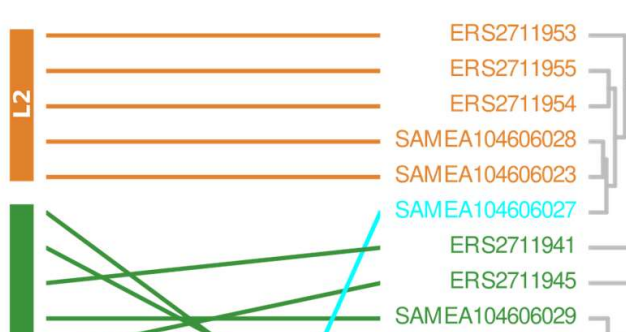

ERS2711941

ERS2711945 SAMEA104606029 SAMEA104606024 ERS2711952 ERS2711950 ERS2711949 ERS2711944 ERS2711940 ERS2711939 ERS2711942 ERS2711947 ERS2711946 ERS2711956 ERS2711948 ERS2711943 SAMEA10460602 SAMEA104606019 SAMEA104606020 ERS271195 SAMEA104606034 SAMEA104606031 SAMEA104606033 SAMEA104606026 SAMEA104606032 SAMEA104606025 SAMEA104606022 SAMEA104606030

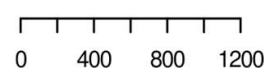

Figure 3. Tanglegram of hierarchically clustered samples.

Clustering was based on IPD and ML phylogeny. Samples are coloured based on lineages. Three samples clustered separately from their lineage. 


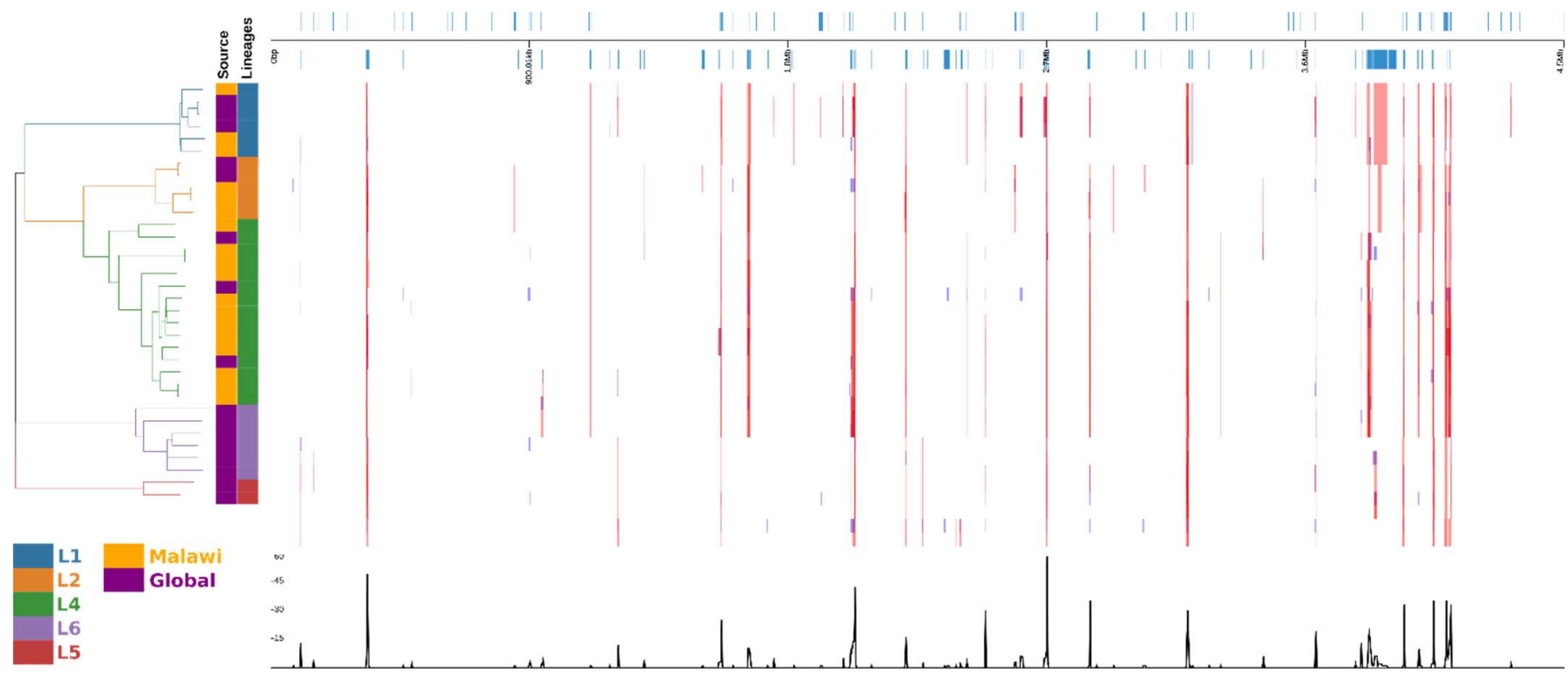

Figure 4. Diverse region in different samples and lineages.

Differences are displayed in alignment frame of the different samples and lineages calculated with default Gubbins parameters. Regions of affected gene locations in the alignment (top). The phylogeny of the 34 samples (left). Recombination events (bottom)

Stability of methylation within Mycobacterium tuberculosis strains.

It was important to study the effect of culture media on methylation patterns. Among our sequences isolates, two (ERS2711943 and ERS2711952) were MGIT grown, the methylation pattern did not appear distinct from the same lineages except "CACGCAG" for ERS2711943 was the lowest in the L1 samples, GATN ${ }_{4}$ RAC was detected in ERS2711943 only. No significant difference could be established between liquid and solid culture isolates for methylated motif CIGGAG (Fishers exact test $p=0.76$ ). As for motif CACGCAG solid cultured isolates were methylated at an average $76 \%$ while liquid cultures were methylated an average $97 \%$. It was found that liquid cultured isolates were significantly more methylated than solid cultures (Fisher's exact $p=0.02$ ) for motif 
CACGCAG. It was observed that this difference was as a result of sample ERS2711943 being lowly methylated at $56 \%$ compared to the rest at $>95 \%$.

We next investigated methylation within the gene regions and promoter regions of genes. Methylation within gene regions ranged between $49 \%$ to $51 \%$ in each strand and there was no over representation of methylation by strand (Chi squared test with Yates correction $\mathrm{P}=0.44)$. On the other hand, methylation within promoter regions of genes ranged from $37 \%$ to $62 \%$ by strand of the promoter methylation. Again there was no significant statistical differences observed by strand (Fishers exact test $P=0.19$ ).

\section{Discussion}

We sequenced 18 genomes of clinical Mtb isolates from Blantyre, Malawi using SMRT sequencing technology and analysed them along with a set of 16 global samples. Studies of Mtb DNA methylation using SMRT sequencing have focused on strains originating from the United States of America (Shell et al., 2013; Zhu et al., 2015), Asia (Zhu et al., 2015) and more recently a small global sample that included Europe, Asia, West Africa and South Africa (J. Phelan et al., 2018). To date no Mtb samples from Malawi or the surrounding region have been subjected to either PacBio SMRT sequencing technology or DNA methylation analysis. In our study, SMRT sequencing of $18 \mathrm{Mtb}$ clinical isolates from Malawi revealed three confidently identified Mtase across the three lineages under study. The activity of these Mtase could be inactivated by three different mutations somewhat in a lineage specific manner. The Mtase MamA was found to be active in all isolates except three L2 (Beijing) isolates putatively courtesy of a point mutation A809C (E270A). This point mutation has been previously characterized (Shell et al., 2013). 
Interestingly, L2 (Beijing) strains have a higher propensity to cause active disease and have been associated with increasing drug resistance in some geographical areas (Cowley et al., 2008; van der Spuy et al., 2009). Whether loss of this Mtase could be associated with success of this organism is an area of interest for future studies. A recent study however failed to establish a possible role of methylation in virulence of Beijing strains (Computational characterisation of DNA methylomes in mycobacterium tuberculosis Beijing hyper- and hypo-virulent strains, n.d.). Similarly, the MamB Mtase (motif CACGCAG) was absent in two (L1) Indo-oceanic isolates. This could be attributed to a C758T (S253L) novel missense mutation recently characterized elsewhere (J. Phelan et al., 2018) and confirmed in this study. While this mutation was putatively found to lead to partial methylation (50-60\%) in a previous study, for the first time, we report that it could also lead to complete loss of Mtase activity as two of our L1 isolates had $0 \%$ methylation. And whether indeed this mutation is responsible for this partial/total loss of methylation now remains debatable. This mutation is present only in EAl6 family of L1 which have been shown to be responsible for recent TB outbreaks globally (Duarte et al., 2017). It is still unknown whether the C758T (S253L) mutation contributes to this transmission. Our investigations as to how the mutation C758T (S253L) could lead to partial loss of methylation in one sample and complete loss in others yielded nothing as we found the rest of the mamB gene to be identical in all the L1 samples including the global samples. There could be yet other unknown mechanisms, possibly a second gene regulating this methylation. In L4 isolates lack of HsdM methylation could be attributed to the C917T (P306L) mutation which was present in 11/12 Malawian isolates. Again lack of methylation was associated with this mutation in all L4 global samples. These results are 
consistent with previous studies which seem to suggest that the P306L mutation is very common in L4 strains (Shell et al., 2013; Zhu et al., 2015). In one study, the mutation was found to be present in 35 out of 37 isolates L4 clinical isolates (Zhu et al., 2015). No cognate restriction enzyme for $H s d M$ has been identified suggesting it could be an orphan Mtase (Zhu et al., 2015). Its principal function could therefore be related to gene regulation rather than restriction modification. Lineage 4 isolates have the highest global prevalence than any other lineage and more studies will be required to establish whether loss of $H s d M$ methylation could be associated with this global success. If indeed HsdM Mtase is disrupted by this mutation in $L 4$, it remains intriguing how some $L 1$ isolates could lose HsdM Mtase in absence of P306L mutation or any other mutation in the $h s d M$ gene. The high frequency of Mtase disrupting mutations in Mtb could be suggestive of a competitive fitness advantage such as immune evasion or even persistence. We found the efficiency of Mtases to be highly variable within and across lineages even in presence of a Mtase gene. The polyketide synthase ( $p k s 15 / 1$ ) locus is responsible for biosynthesis of phenolic glycolipid (PGL), a cell wall component (Caws et al., 2008; Reed et al., 2004) and has widely been used to discriminate between L4 isolates against L1 and L2 isolates owing to a 7 bp deletion in L4 isolates (Caws et al., 2008; Gagneux \& Small, 2007). In this study for the first time, we have demonstrated the potential of using the $p k s 15 / 1$ locus to classify L5 isolates using 9bp (CGGTGCTGG) deletion a distinct substitution A50G and an insertion GGGCCGC while L6 isolates could also be classified using a 6bp (GGGCCGC) at the same position of the $7 \mathrm{bp}$ deletion in L4 isolates. The pks15/1 locus therefore could be a valuable marker for identifying isolates belonging to $L 5$ and $L 6$. The large number of genomic re-arrangements observed in mostly cell wall component genes PPE, PE-PGRS 
and ESAT- 6 is evidence of the large variations that exist among different strains and lineages of $M t b$ in responding to host immunity.

We believe the complete characterization of DNA methylation in Mtb could help provide clues to some of the clinical phenotypes which have been associated with strain and lineage variation. In this study no compelling correlation could be established between methylation and Mtb growth condition although MGIT cultures were shown to sequence at a slightly lower coverage. Overall data presented in this study shows the potential of SMRT sequencing long reads to help us better understand the complete biology of Mycobacterium tuberculosis by resolving difficult regions of the genome and elucidating the complete methylome of the pathogen. This study could not establish the direct association between mutations and loss of Mtase activity and also why some samples could show low levels of Mtase activity than others. To better understand the complete impact of DNA methylation within specific strains and lineages, subsequent studies will need to integrate transcriptomic and proteomic data to methylomes.

\section{Materials and methods}

\section{Sample collection}

310 Frozen archived clinical isolates from a previous prospective cohort study, Studying Persistence and Understanding Tuberculosis in Malawi (S.P.U.T.U.M) (Sloan et al., 2015) were characterized. These were from patients aged 16-65 years old presenting with bacteriologically culture confirmed pulmonary Mtb between June 2010 and December 
positive isolates, 18 were selected based on which isolates were the first to be successfully revived from frozen state and used in this study.

\section{Bacterial growth conditions}

All experiments involving Mtb were performed in a Biosafety Level (BSL) 3 Laboratory, University of Malawi-College of Medicine/ Malawi Liverpool Welcome Trust (CoM/MLW) TB laboratory and at Liverpool School of Tropical Medicine following Standard Operating Procedures (SOPs). All reagents used were from Sigma-Aldrich unless otherwise stated. For liquid culture, strains were grown in Middlebrook 7H9 broth base supplemented with oleic acid, albumin, dextrose and catalase (OADC) and an antibiotic mixture of polymyxin B, amphotericin B, nalidixic acid, trimethoprim and azlocillin (PANTA). Tubes were incubated in a BACTEC MGIT 960 instrument at $37^{\circ} \mathrm{C}$ and monitored once a week for possible growth for up to eight weeks. Isolates used in the study were from a previous study for which ethics approval had previously been granted by the College of Medicine Ethics Committee (COMREC), University of Malawi (Sloan et al., 2015). Solid culture inoculation was done on Lowenstein-Jensen (LJ) slopes following laboratory SOP. Cultures were grown to mid-log phase and harvested at $\sim 7$ th week and used for DNA isolation. Mtb was confirmed using both the BD MGIT TBC ID test device (Becton

333 Dickinson, Maryland U.S.A) following manufacturer's instructions and Ziehl Neelsen (ZN) staining for acid fast bacilli (AFB). 


\section{DNA Extraction}

Genomic DNA was isolated using the traditional Cetyltrimethylammonium bromide (CTAB) method as previously described (Somerville et al., 2005). Extracted DNA was quantified using Qubit 3.0 fluorometer (Life Technologies, USA) according to manufacturer's instructions and DNA purity was determined on a NanoDrop ND-1000 Spectrophotometer V3.7 (Thermo Scientific, Wilmington U.S.A) following manufacturer's instructions. DNA purity was checked at absorbance $260 \mathrm{~nm}$ and $280 \mathrm{~nm}$ by calculating a ratio of A260/A280. DNA quality was analyzed on 1.5\% Agarose Gel electrophoresis and visualized under UV light following ethidium bromide staining.

\section{Genotyping of Mtb Isolates}

Genotyping of isolates was done at the Liverpool School of Tropical Medicine, United Kingdom. Lineage specific deletions were detected using a singleplex PCR based method with specific oligonucleotide primers targeting the regions of difference RD239, RD105 and RD750. PCR reactions were performed as documented in our previous publication (Ndhlovu et al., 2019).

\section{DNA Sequencing}

Purified genomic DNA libraries were sequenced at the Centre for Genomic Research (CGR), Institute of Integrative Biology, University of Liverpool, United Kingdom. DNA libraries were purified with $1 \mathrm{x}$ cleaned AMPure beads (Agencourt) and the quantity and quality was assessed using the Qubit and NanoDrop assays respectively. In addition, the Fragment Analyzer using a high sensitivity genomic kit (Advanced Analytical Technologies, Inc.) was used to determine the average size of the DNA and the extent of degradation. DNA was treated with Exonuclease $\mathrm{V} 11$ at $37^{\circ} \mathrm{C}$ for 15 minutes. The ends 
of the DNA were repaired as described by the manufacturer (Pacific Biosciences, Menlo Park, CA, USA). The sample was incubated for 20 minutes at $37^{\circ} \mathrm{C}$ with DNA damage repair mix supplied in the SMRTbell library kit (Pac Bio). This was followed by a 5-minute incubation at $25^{\circ} \mathrm{C}$ with end repair mix. DNA was cleaned using $0.5 x$ AMPure and $70 \%$ ethanol washes. DNA was ligated to adapter overnight at $25^{\circ} \mathrm{C}$. Ligation was terminated by incubation at $65^{\circ} \mathrm{C}$ for 10 minutes followed by exonuclease treatment for 1 hour at $37^{\circ} \mathrm{C}$. The SMRTbell library was purified with $0.5 x$ AMPure beads. The library was size selected with $0.75 \%$ blue pippin cassettes in the range $7000-20000 \mathrm{bp}$. The recovered fragments were damage repaired again. The quantity of library and therefore the recovery was determined by Qubit assay and the average fragment size determined by Fragment Analyzer. SMRTbell library was annealed to sequencing primer at values predetermined by the Binding Calculator (PacBio) and a complex made with the DNA polymerase (P6/C4 chemistry). The complex was bound to Magbeads and this was used to set up the required number of SMRT cells for the project (two for each sample). Sequencing was performed on Pacific Biosciences RSII sequencing system (Pacific Biosciences, Menlo Park, CA, USA) using 360-minute movie times per cell, yielding 300x average genome coverage. The generated data have been submitted to the ENA databases (Bio-Project:

\section{PRJEB28592).}

\section{Bioinformatics Analysis}

Generated long Pacbio reads were analysed using the RS_Modification_and_Motif_Analysis.1 protocol as part of SMRT analysis in SMRT Portal (version 2.2.0). To increase the robustness of our analysis, we included previously published Mtb methylation study Pacbio data (Bio-project: PRJEB21888) (J. Phelan et 
al., 2018) and conducted both genomic and methylation comparisons of the two datasets. Although Bio-project PRJEB21888 had 18 genomes, we could only access 16 and these were used in our analysis. However, we evaluated PRJEB21888 sequences using SMRT Portal (version 5.1.0). Reads were mapped using the Basic Local Alignment with Successive Refinement (BLASR) (Chaisson \& Tesler, 2012) algorithm within the SMRT portal. Strain specific genomes were generated by mapping the reads to the reference genome (H37Rv) using Quiver tool. Standard settings were used to detect base modifications and methylation motifs in the strain's genome. Inter-pulse duration (IPD) ratio (observed vs expected) was measured for the modification detection (Zhu et al., 2015). Computational validation of our samples' lineages and lineage identification of PRJEB21888 samples were done using TB-Profiler (Jody E Phelan et al., n.d.). Comparative analysis of pks15 (Rv2947c) gene was used to report lineages of the samples specifically those from PRJEB21888. The MAFFT (version 7.310) (Katoh \& Standley, 2013) was used to generate multiple sequence alignment of consensus sequences against $H 37 R v$ reference. Following removal of the reference from the alignment, maximum likelihood (ML) phylogeny was constructed for the remaining sequences using RaxML (v8.2.12) GTR $+\Gamma$ model (Stamatakis, 2014) applying 1000 bootstrap iterations. Although Mtb has a highly rigid and non-recombinogenic genome (>99\% nucleotide identity), to report diverse genomic regions among isolates, Gubbins (2.4.1) (Croucher et al., 2015) was applied with the default parameters over previously generated alignment of 34 genomes and earlier constructed ML phylogeny as an initial tree. Identified recombination hot spots were plotted with phylogeny generated without 
404

405

hot spots, affected genes details and the metadata using Phandango (Hadfield et al., 2018). Samples were clustered hierarchically based on m6A IPD ratio pattern.

Multiple sequence alignment of Mtase genes ( $m a m A, \operatorname{mamB}, h s d M$ and $h s d S$ ) sequences against the reference gene from H37Rv genome was used to identify possible mutations responsible for loss of methylation. Comparative analysis of well characterized methylation sites among samples were performed. Clustering of the samples based on their reported IPD ratios at methylated sites was performed and compared with clustering in ML phylogeny.

\section{Acknowledgements}

We thank the guardians and patients who participated in this study, and the staff at Queen

Elizabeth Central Hospital for their assistance.

\section{Competing Interests}

The authors declare no interest

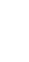




\section{References}

Albanna, A. S., Reed, M. B., Kotar, K. V, Fallow, A., Mclntosh, F. A., Behr, M. A., \& Menzies, D. (2011). Reduced transmissibility of East African Indian strains of Mycobacterium tuberculosis. PloS One, 6(9), e25075. https://doi.org/10.1371/journal.pone.0025075 [doi]

Balaban, N. Q., Merrin, J., Chait, R., Kowalik, L., \& Leibler, S. (2004). Bacterial Persistence as a Phenotypic Switch. Science, 305(5690), 1622-1625. https://doi.org/10.1126/science.1099390

Brennan, M. J., \& Delogu, G. (2002). The PE multigene family: a 'molecular mantra' for mycobacteria. Trends in Microbiology, 10(5), 246-249. https://doi.org/http://dx.doi.org/10.1016/S0966-842X(02)02335-1

Casadesus, J., \& Low, D. (2006). Epigenetic gene regulation in the bacterial world. Microbiology and Molecular Biology Reviews : MMBR, 70(3), 830-856. https://doi.org/70/3/830 [pii]

Caws, M., Thwaites, G., Dunstan, S., Hawn, T. R., Lan, N. T., Thuong, N. T., Stepniewska, K., Huyen, M. N., Bang, N. D., Loc, T. H., Gagneux, S., van Soolingen, D., Kremer, K., van der Sande, M., Small, P., Anh, P. T., Chinh, N. T., Quy, H. T., Duyen, N. T., ... Farrar, J. (2008). The influence of host and bacterial genotype on the development of disseminated disease with Mycobacterium tuberculosis. PLoS Pathogens, 4(3), e1000034. https://doi.org/10.1371/journal.ppat.1000034 [doi]

Chaisson, M. J., \& Tesler, G. (2012). Mapping single molecule sequencing reads using basic local alignment with successive refinement (BLASR): application and theory. BMC Bioinformatics, 13, 238. https://doi.org/10.1186/1471-2105-13-238 [doi] 
Cole, S. T. (1999). Learning from the genome sequence of Mycobacterium tuberculosis H37Rv. FEBS Letters, 452(1-2), 7-10. https://doi.org/http://dx.doi.org/10.1016/S0014-5793(99)00536-0

Cole, S. T. (2002). Comparative and functional genomics of the Mycobacterium tuberculosis complex. Microbiology, 148(10), 2919-2928.

Computational characterisation of DNA methylomes in mycobacterium tuberculosis Beijing hyper- and hypo-virulent strains. (n.d.). Retrieved April 10, 2020, from https://etd.uwc.ac.za/xmlui/handle/11394/4756

Constant, P., Perez, E., Malaga, W., Laneelle, M., Saurel, O., Daffe, M., \& Daffe, M. (2002). Role of pks15/1 gene in the Biosynthesis of Phenoglycolipids in the Mycobacterium tuberculosis complex. EVIDENCE THAT ALL STRAINS SYNTHESIZE GLYCOSYLATED p-HYDROXYBENZOIC METHYL ESTERS AND THAT STRAINS DEVOID OF PHENOLGLYCOLIPIDS HABOUR A FRAMESHIFT MUTAT. Journal of Biological Chemistry, 227, 38148-38158.

Cowley, D., Govender, D., February, B., Wolfe, M., Steyn, L., Evans, J., Wilkinson, R. J., \& Nicol, M. P. (2008). Recent and rapid emergence of W-Beijing strains of Mycobacterium tuberculosis in Cape Town, South Africa. Clinical Infectious Diseases : An Official Publication of the Infectious Diseases Society of America, 47(10), 1252-1259. https://doi.org/10.1086/592575 [doi]

Croucher, N. J., Page, A. J., Connor, T. R., Delaney, A. J., Keane, J. A., Bentley, S. D., Parkhill, J., \& Harris, S. R. (2015). Rapid phylogenetic analysis of large samples of recombinant bacterial whole genome sequences using Gubbins. Nucleic Acids Research. https://doi.org/10.1093/nar/gku1196 
Davies, P., Barnes, P., \& Gordon, S. (Eds.). (2014). Clinical Tuberculosis (5th ed.). CRC Press.

De Schacht, C., Mutaquiha, C., Faria, F., Castro, G., Manaca, N., Manhiça, I., \& Cowan, J. (2019). Barriers to access and adherence to tuberculosis services, as perceived by patients: A qualitative study in Mozambique. PLoS ONE, 14(7). https://doi.org/10.1371/journal.pone.0219470

Duarte, T. A., Nery, J. S., Boechat, N., Pereira, S. M., Simonsen, V., Oliveira, M., Gomes, M. G. M., Penha-Goncalves, C., Barreto, M. L., \& Barbosa, T. (2017). A systematic review of East African-Indian family of Mycobacterium tuberculosis in Brazil. The Brazilian Journal of Infectious Diseases : An Official Publication of the Brazilian Society of Infectious Diseases, 21(3), 317-324. https://doi.org/S14138670(16)30547-5 [pii]

Filliol, I., Motiwala, A. S., Cavatore, M., Qi, W., Hazbón, M. H., del Valle, M. B., Fyfe, J., García-García, L., Rastogi, N., Sola, C., Zozio, T., Guerrero, M. I., León, C. I., Crabtree, J., Angiuoli, S., Eisenach, K. D., Durmaz, R., Joloba, M. L., Rendón, A., ... Alland, D. (2006). Global Phylogeny of Mycobacterium tuberculosis Based on Single Nucleotide Polymorphism (SNP) Analysis: Insights into Tuberculosis Evolution, Phylogenetic Accuracy of Other DNA Fingerprinting Systems, and Recommendations for a Minimal Standard SNP Set. Journal of Bacteriology, 188(2), 759-772. https://doi.org/10.1128/JB.188.2.759-772.2006

Fishbein, S., van Wyk, N., Warren, R. M., \& Sampson, S. L. (2015). Phylogeny to function: PE/PPE protein evolution and impact on Mycobacterium tuberculosis pathogenicity. Molecular Microbiology, 96(5), 901-916. 
https://doi.org/10.1111/mmi.12981 [doi]

Gagneux, S., \& Small, P. M. (2007). Global phylogeography of Mycobacterium tuberculosis and implications for tuberculosis product development. The Lancet.Infectious Diseases, 7(5), 328-337. https://doi.org/S1473-3099(07)70108-1 [pii]

Grover, S., Sharma, T., Singh, Y., Kohli, S., Manjunath, P., Singh, A., Wieler, L. H., Tedin, K., Ehtesham, N. Z., Hasnain, S. E., \& Semmler, T. (2018). The PGRS domain of Mycobacterium tuberculosis PE_PGRS protein Rv0297 is involved in Endoplasmic reticulum stress-mediated apoptosis through toll-like receptor 4. MBio, 9(3). https://doi.org/10.1128/mBio.01017-18

Hadfield, J., Croucher, N. J., Goater, R. J., Abudahab, K., Aanensen, D. M., \& Harris, S. R. (2018). Phandango: An interactive viewer for bacterial population genomics. Bioinformatics. https://doi.org/10.1093/bioinformatics/btx610

Hershberg, R., Lipatov, M., Small, P. M., Sheffer, H., Niemann, S., Homolka, S., Roach, J. C., Kremer, K., Petrov, D. A., Feldman, M. W., \& Gagneux, S. (2008). High functional diversity in Mycobacterium tuberculosis driven by genetic drift and human demography. PLoS Biology, 6(12), e311. https://doi.org/10.1371/journal.pbio.0060311 [doi]

Katoh, K., \& Standley, D. M. (2013). MAFFT multiple sequence alignment software version 7: improvements in performance and usability. Molecular Biology and Evolution, 30(4), 772-780. https://doi.org/10.1093/molbev/mst010 [doi]

Ndhlovu, V., Kiran, A., Sloan, D., Mandala, W., Kontogianni, K., Kamdolozi, M., Caws, M., \& Davies, G. (2019). Genetic diversity of Mycobacterium tuberculosis clinical 
isolates in Blantyre, Malawi. Heliyon, 5(10).

Phelan, J., de Sessions, P. F., Tientcheu, L., Perdigao, J., Machado, D., Hasan, R., Hasan, Z., Bergval, I. L., Anthony, R., McNerney, R., Antonio, M., Portugal, I., Viveiros, M., Campino, S., Hibberd, M. L., \& Clark, T. G. (2018). Methylation in Mycobacterium tuberculosis is lineage specific with associated mutations present globally. Scientific Reports, 8(1), 160-017-18188-y. https://doi.org/10.1038/s41598017-18188-y [doi]

Phelan, J E, Coll, F., Bergval, I., Anthony, R. M., Warren, R., Sampson, S. L., van

Phelan, Jody E, O’sullivan, D. M., Machado, D., Ramos, J., Oppong, Y. E. A., Campino, S., O’grady, J., Mcnerney, R., Hibberd, M. L., Viveiros, M., Huggett, J. F., \& Clark, T. G. (n.d.). Integrating informatics tools and portable sequencing technology for rapid detection of resistance to anti-tuberculous drugs. https://doi.org/10.1186/s13073-019-0650-x

Reed, M. B., Domenech, P., Manca, C., Su, H., Barczak, A. K., Kreiswirth, B. N., Kaplan, G., \& 3rd, C. E. B. (2004). A glycolipid of hypervirulent tuberculosis strains that inhibits the innate immune response. Nature, 431(7004), 84-87. https://doi.org/10.1038/nature02837 [doi] 
Shell, S. S., Prestwich, E. G., Baek, S. H., Shah, R. R., Sassetti, C. M., Dedon, P. C., \& Fortune, S. M. (2013). DNA methylation impacts gene expression and ensures hypoxic survival of Mycobacterium tuberculosis. PLoS Pathogens, 9(7), e1003419. https://doi.org/10.1371/journal.ppat.1003419 [doi]

Sloan, D. J., Mwandumba, H. C., Garton, N. J., Khoo, S. H., Butterworth, A. E., Allain, T. J., Heyderman, R. S., Corbett, E. L., Barer, M. R., \& Davies, G. R. (2015). Pharmacodynamic Modeling of Bacillary Elimination Rates and Detection of Bacterial Lipid Bodies in Sputum to Predict and Understand Outcomes in Treatment of Pulmonary Tuberculosis. Clinical Infectious Diseases : An Official Publication of the Infectious Diseases Society of America, 61(1), 1-8. https://doi.org/10.1093/cid/civ195 [doi]

Somerville, W., Thibert, L., Schwartzman, K., \& Behr, M. A. (2005). Extraction of Mycobacterium tuberculosis DNA: a Question of Containment. Journal of Clinical Microbiology, 43(6), 2996-2997. https://doi.org/10.1128/JCM.43.6.2996-2997.2005

Stamatakis, A. (2014). RAxML version 8: a tool for phylogenetic analysis and postanalysis of large phylogenies. Bioinformatics (Oxford, England), 30(9), 1312-1313. https://doi.org/10.1093/bioinformatics/btu033 [doi]

van der Spuy, G. D., Kremer, K., Ndabambi, S. L., Beyers, N., Dunbar, R., Marais, B. J., van Helden, P. D., \& Warren, R. M. (2009). Changing Mycobacterium tuberculosis population highlights clade-specific pathogenic characteristics. Tuberculosis (Edinburgh, Scotland), 89(2), 120-125. https://doi.org/10.1016/j.tube.2008.09.003 [doi]

WHO. (2020). WHO | Global tuberculosis report 2019. WHO. 
564 Zhu, L., Zhong, J., Jia, X., Liu, G., Kang, Y., Dong, M., Zhang, X., Li, Q., Yue, L., Li, C.,

565 Fu, J., Xiao, J., Yan, J., Zhang, B., Lei, M., Chen, S., Lv, L., Zhu, B., Huang, H., \&

566 Chen, F. (2015). Precision methylome characterization of Mycobacterium

567 tuberculosis complex (MTBC) using PacBio single-molecule real-time (SMRT)

568 technology. Nucleic Acids Research, 44(2), 730-743.

569 https://doi.org/10.1093/nar/gkv1498

\section{Supplementary files}

571 Supplementary Table 1. Methylation efficiency for 34 Mycobacterium tuberculosis

572 samples 